\title{
PREPARING LAW STUDENTS FOR PRACTICE: A NON-VERBAL DEVELOPMENTAL SKILL APPROACH
}

\section{$1 \quad$ Introduction}

"A skilful trial lawyer or litigator cannot be successful without mastering the ability to decode nonverbal behaviour". (Nierenberg and Calero How to Read a Person like a Book (1980) 58).

The debate about whether law schools in South Africa prepare students adequately for practice has been ongoing for years now and the nature of legal education has been called into question many times in the past few years. The disjuncture between legal education and professional demands has been echoed by many professional bodies in South Africa and there is general consensus that there is an urgent need to produce well-rounded and better-educated law graduates who are ready for practice (Van Niekerk "The Four Year Undergraduate LLB: Where to From Here?" 2013 34(3) Obiter 533). It goes without saying that the legacy of apartheid and the dehumanising effect of a racialised past has seen many students entering tertiary education from a poor and imbalanced schooling system and this has served as a major obstacle to success (Greenbaum "Re-Visioning Legal Education in South Africa: Harmonising the Aspirations of Transformative Constitutionalism with the Challenges of Our Educational Legacy" (8 November 2014) https://ssrn.com/abstract=2575289 (accessed 2018-0210)). For many law schools in South Africa, imparting skills that are essential for lawyering in the "new" South Africa remains a challenge - one that will only be met once the effects of inequality, segregation and a socio-economic divide have been fully addressed. These factors cannot be addressed overnight, but we can immediately address the need to equip students with basic lawyering skills necessary for practice and success in the outside world. South Africa is a diverse, multicultural country with 11 official languages and it is imperative that future lawyers are able to provide a service effectively to clients from varied backgrounds, including ones dissimilar to their own.

Key to effective lawyering are good communication skills and it goes without saying that the legal profession places huge emphasis on oral and written communication, which are important lawyering skills (Barkai "Nonverbal Communication From the Other Side: Speaking Body Language" 1990 San Diego Law Review 101). It is often said that "communication is the tool of trade" for lawyers as they are expected to communicate effectively on a daily basis with a wide range of clients and in a variety of settings. While speech and written text are crucial skills for effective lawyering, one should acknowledge that words are not the only essential component of the communication process (Mandal "Nonverbal Communication in Humans" 
2014 Journal of Human Behaviour in the Social Environment 417-421). In fact, studies have shown that over 90 per cent of the meaning of a message takes place non-verbally, and that non-verbal communication is at least as, important as, if not more so, than the verbal component (Mehrabian Nonverbal Communication (1972) 52-55). Considering that the legal profession in South Africa, as well as worldwide, places huge emphasis on communication, it is surprising that there has been little scholarship concerning non-verbal skill development in law. A key objective of this article is to highlight the fact that non-verbal skills development is crucial to understanding law at all levels and to producing graduates who are ready for practice. The article will commence with a brief overview of non-verbal communication, analyse its characteristics, and then move onto its importance to the legal sector (more specifically in legal practice) and finally will focus on the use of non-verbal communication in the classroom. The article seeks to address the grey area that currently exists within the current legal framework with the objective of introducing non-verbal practice into law curriculums in South Africa. As a way forward, it will become evident that there is a strong need for robust practical and skills emphasis in most law programmes, and such interrogation should ultimately prepare graduates for practice. The study of non-verbal communication amalgamated with traditional legal skills would go a long way to producing law graduates who have both a systematic and coherent body of knowledge, and a high level of cognitive and generic skills; together these will pave their way for successful practice.

\section{Understanding non-verbal communication}

According to John Barkai, non-verbal communication has been evident for centuries as reflected in the many references to non-verbal communication in the Bible. However, it has only truly become an area of intensive scientific research in the last two to three decades (Barkai 1990 San Diego Law Review 101). The six different disciplines that have contributed to research in the field in recent times include anthropology, linguistics, psychoanalysis, clinical psychology, social psychology and sociology (Barkai 1990 San Diego Law Review 101). However, in law, there has been limited research done on the role of non-verbal communication (Brayne "Counselling Skills for the Lawyer: Can the Lawyer Learn Anything from the Counsellors?" 199832 The Law Teacher 137). A study of legal literature suggests that what little research there has been into non-verbal communication in law has focused on the courtroom environment and body language (Barkai 1990 San Diego Law Review 101). Considering that the legal profession places great emphasis on communication, it is difficult to fathom that so little research has been done on the impact of non-verbal communication in the legal sector (Barkai 1990 San Diego Law Review 101).

Effective communication involves an exploration of the different communication channels, and consideration of the message type, environment and audience (Westmayer, DiCioccio and Rubin "Appropriateness and Effectiveness of Communication Channels in Competent Interpersonal Communication" 199848 Journal of Communication 27). The ability to explore various channels of 
communication is key to developing versatility as a communicator (Westmayer et al 1998 Journal of Communication 27).

Communication generally occurs in a verbal form, a non-verbal form or a combination of both (Duke "Nonverbal Behaviour and the Communication Process" 197425 College Composition and Communication 397-399). While the use of words is integral to verbal communication, non-verbal communication involves communication devoid of words (Knapp and Hall Nonverbal Communication in Human Interaction (2002) 108). The characteristics and features of non-verbal communication are endless but common forms of non-verbal communication involve touch, smell, body language, posturing, facial expressions and even silence, among others (Bull "State of the Art: Nonverbal Communication" 200114 The Psychologist 644).

Early studies by Mehrabian on non-verbal communication attached relative values to the different constituents of communication. Interestingly, he assessed the impact of the non-verbal component on interpersonal communication to be as high as 93 per cent (Mehrabian Nonverbal Communication (1972) 52-55; see also article by Park and Park "Correlation between Nonverbal Communication and Objective Structured Clinical Examination Score in Medical Students" 2018 Korean Journal of Medical Education 200 for a discussion on the impact of non-verbal communication on the communication process). Although studies on non-verbal communication have reached slightly varying figures in terms of impact, what is common to most studies is the dominant nature of non-verbal communication in the interpersonal communicative process (Feldman Application of Nonverbal Behavioral Theories and Research (1992) 84).

A fascinating aspect of non-verbal communication (unlike words or language) is that it is not easily controlled (Milford "Nonverbal Communication" 200127 Litigation 33). Facial expressions, body language and gestures are not as easily manipulated as words since they are coordinated by feelings and emotions (Milford 2001 Litigation 33). The spectrum of components that inform non-verbal behaviour is endless and the various constituents such as body patterns, facial expressions, gestures and postures convey a plethora of meaning and messages (Milford 2001 Litigation 33). A database of behavioural, non-verbal patterns is activated when humans communicate and these involvement cues or patterns serve as a rich source of information (Coker and Burgoon "The Nature of Conversational Involvement and Nonverbal Coding Patterns" 198713 Human Communication Research 463). Non-verbal communication provides an indicator of people's emotions and delivers vital information about a person's intellect, true self and their credibility (Coker and Burgoon 1987 Human Communication Research 463). As part of the communication process, involvement cues and behavioural patterns serve as an important source of information (Coker and Burgoon 1987 Human Communication Research 463). A person's actions, speech or even silence are part of a communication process that conveys messages to others about ourselves (Coker and Burgoon 1987 Human Communication Research 463). 


\section{Characteristics of non-verbal communication}

According to Kundu, four key characteristics of non-verbal communication are significant within the educational context (Kundu "Visual Literacy: Teaching Non-Verbal Communication Through Television" 197616 Educational Technology 31-33).

The first is that non-verbal communication conveys meaning about a communicator's present state or attitude, irrespective of when an event being described took place. In other words, non-verbal communication reflects the communicator's state of mind at the time that a message is being conveyed.

The second characteristic is that non-verbal communication depicts the kind of relationship that communicators share (Kundu 1976 Educational Technology 31-33). The handshake, which is regarded as a universal "warm gesture", generally establishes a good rapport with another and is usually seen as the first step to creating a trusting environment (Kirch "Non-Verbal Communication Across Cultures" 197963 The Modern Language Journal 416-423).

The third characteristic is that non-verbal communication can only transfer meaning in fixed or stable ways (Kundu 1976 Educational Technology 31-33). According to Ekman, the human body leaks information without full awareness of such leakage at most times (Ekman Emotion in the Human Face (1982) 75). The face is said to be the central communicator of emotion; anger, sadness, happiness, fear, surprise and disgust are communicated through eye movement and facial expressions (Ekman Emotion in the Human Face 75). Gestures, postures and different forms of body movement convey meaning in different forms and, at times, serve as cues to continue or conclude an interaction (Ekman Emotion in the Human Face 75).

The fourth characteristic is that the meaning of non-verbal signs (unlike words) is not fixed but is dependent on a multitude of factors, such as the verbal environment, context and culture and the person (Kundu 1976 Educational Technology 31-33). As South Africa has a diverse set of cultures, it is important for an attorney to be cognisant of the fact that culture, together with other factors, may have an effect on the evaluation of nonverbal actions. Work believed that all communication is cross-cultural and a person's own cultural system ought to be taken into account when interacting with another (Work "ERIC Report: Communication across Cultures" 198130 Communication Education 27-36). A typical example of potential cross cultural misunderstanding would be the late arrival of a client due to the client being polychronic rather than insolent. It is therefore critical to be wary of a variance of factors affecting meaning when analysing nonverbal communication.

\section{The importance of non-verbal communication in developing good lawyering skills}

According to Kevelson, non-verbal signs convey a multitude of messages and serve different functions (Kevelson The Law as a System of Signs 
(1988) 5). For any young attorney, the ability to perceive and analyse nonverbal signs within a legal environment is crucial to developing good lawyering skills (Balkin "The Promise of Legal Semiotics" (1991) Faculty Scholarship Series Paper 278 http://digitalcommons.law.yale.edu/fsspapers/278 (accessed 2018-03-26) 18).

Non-verbal communication in the form of personal appearance, body language, vocal cues, facial expressions, personal space and posture play important roles in obtaining relevant and accurate information from a client and deserves an attorney's conscious attention (Kevelson The Law as a System of Signs 5). Within a legal context, non-verbal communication allows for: the creation of good first impressions; manipulation and persuasion; the stereotyping of others; the sharing of conscious and unconscious feelings, beliefs and attitudes; aiding speech production; and decoding deception (Burgoon, Buller and Woodall Nonverbal Communication: The Unspoken Dialogue (1989) 27). At the start of a consultation, establishing a good rapport with a client through the use of warm gestures such as a handshake or a smile can impact on the success of a case (Burgoon et al Nonverbal Communication 27). Getting the client to confide in a stranger is critical to a productive consultation process and this can only be achieved through establishing an enabling environment. An understanding of non-verbal communication is therefore vital to establishing good interactive skills (Balkin http://digitalcommons.law.yale.edu/fss-papers/278).

The importance of using non-verbal communication in a courtroom scenario cannot be underemphasised. LeVan ("Non-Verbal Communication in the Courtroom: Attorneys Beware" 19848 Law and Psychology Review 83-104) underlined the significance of non-verbal communication within the setting of a formal courtroom when he stated:

"In the courtroom, nonverbal communication subtly affects the entire proceedings of a trial. It is constantly present and being asserted, yet the attorney is often unaware of its existence."

The use of non-verbal techniques such as dress, gestures and body language, combined with other components such as pitch and tone, can be crucial to establishing a good affinity with jurors and judges (LeVan 1984 Law and Psychology Review 83-104). Whereas the use of "reinforcing" cues such as gaze and smiles can have a positive influence on court officials, negative non-verbal cues such as lack of eye contact or antagonistic gestures can have the opposite effect (Balkin http://digitalcommons.law.yale.edu/fss-papers/278).

Matlon contends that the ability to decipher variances between the verbal and non-verbal interface is crucial to the effective interrogation of a witness (Matlon Communication in the Legal Process (1988) 82). Hostile cues on the part of a witness, such as fidgety movements, increased tone and lack of eye contact, points to uneasiness and possible deception (Matlon Communication in the Legal Process 82). There is little doubt that the ability to cross-examine a witness effectively or attain useful information from that witness depends largely on the use of non-verbal cues such as measured eye contact, inspired facial expressions, inventive body positioning, varied tone of voice and apt head nods, among others (Balkin 
http://digitalcommons.law.yale.edu/fss-papers/278). The ability to exercise conscious control over the non-verbal is not as easy as exercising control over words; hence, a skilled attorney would look out for any inconsistencies between the two when looking to "break down" a witness in a courtroom setting (Remland "The Implicit Ad Hominem Fallacy: Nonverbal Displays of Status in Argumentative Discourse" 198419 Journal of the American Forensics Association 71-77). Similarly, observing the facial expressions, gaze patterns and body movements of the presiding officer or judge can provide useful hints as to whether such questioning has reached saturation point and whether the attorney should continue with the line of questioning or move on (Remland 1984 Journal of the American Forensics Association 71-77).

In adversarial settings such as those adopted in South African courts, the presiding officer quite often makes a finding based on the credibility of the various witnesses who have testified in court and such credibility findings often factor in the witnesses' demeanour, body language and testimony during a trial (Stellenbosch Farmer's Winery Group Ltd v Martell et Cie 2003 (1) SA 11 (SCA)). The significance of non-verbal communication in a formal courtroom setting cannot be overlooked.

In a study conducted by lyer, a purposive sampling approach was used to select and interview attorneys from the Empangeni Justice Centre to ascertain their views on the importance of non-verbal communication within the domain of the legal environment (Iyer The Semiotics of Non-Verbal Communication in the Attorney, Client Consultation Process (doctoral thesis, University of Zululand) 2011 105). It was interesting to note that all participants in the study saw the need to train law students in the art of nonverbal communication early in their academic studies (lyer The Semiotics of Non-Verbal Communication 105). The participants studied law at various institutions in South Africa and most felt that they received inadequate training in the non-verbal component of communication, which was extremely important in practice. This, according to most of the participants, was a major reason that many of them struggled to gain the trust and confidence of clients during the consultation process and likewise many clients refused to "open up to them" on a professional level (lyer The Semiotics of Non-Verbal Communication 105). The majority of participants reiterated the view that an attorney could obtain more information from clients and become better communicators by using non-verbal communication in different legal settings (lyer The Semiotics of Non-Verbal Communication 105). All participants expressed the desire to understand and decode non-verbal communication in an effort to improve their legal communication skills (lyer The Semiotics of Non-Verbal Communication 105). The study clearly indicated that non-verbal communication has a vital role to play in improving the legal communication skills of the legal practitioner.

In the legal profession, deception is a topic spoken of quite often and nonverbal communication has a role to play in identifying such conduct. The ability to alter the quantity, quality and clarity of a verbal message at similar or different intervals constitutes deception. Although some people are able to tell a lie better than others, it is not easy to submerge the subconscious 
mind, which generally acts contrary to the verbal lie (Rugbeer Deceptive Communication: When It Is Legitimate to Deceive Others, and When It Is Not (doctoral thesis, University of Zululand) 2005 83). Studies have shown that although a trained liar attempts to suppress certain gestures when lying, other micro-gestures, such as regular twitching, perspiration, systematic blinking, flushed cheeks, the touching of the nose or mouth and dilation of the pupil, serve as indicators of deception (Pease Body Language (1988) 57).

The ability of an attorney to detect deception during an initial consultation and during court proceedings can be critical to the success of a case. It is only through careful observation as well as training and practice that the subconscious non-verbal signs become evident to the naked eye (Ekman and Friesman "Detecting Deception of the Body or Face" 197429 Journal of Personality and Social Psychology 33). Determining when someone is lying is a difficult process. One cannot simply rely on the more obvious sources such as the face and head; it is essential also to focus on the leg, arm and foot movements, which may display erratic movement at times (Duke 1974 College Composition and Communication 401). In addition, a person who intentionally wishes to convey false information and messages may position himself/herself further away from the listener than when speaking the truth, may gesticulate less frequently, and decrease the number of head nods (Mehrabian Nonverbal Communication 52-55). In addition, studies have shown that her speech rate may become slower, speech errors are made more regularly and the tendency to smile more becomes apparent compared to when the truth is being spoken (Mehrabian Nonverbal Communication 52-55). Although one has to be wary of misreading feelings of anxiety, trepidation and cultural mannerisms as indicators of deception, studies have shown that even the most skillful liar eventually releases a cluster of nonverbal signals that tend to contradict the verbal message and any competent attorney must be in a position to detect such contradictions (Ekman and Friesman 1974 Journal of Personality and Social Psychology 33). A mastery of non-verbal communication is therefore crucial to developing good lawyering skills. These skills must be developed as early as possible with the law teacher being aware of the influences of non-verbal behaviours in the classroom as well as outside the classroom.

\section{Introducing non-verbal communication in the classroom}

The earlier sections highlighted the importance of developing non-verbal skills in the law graduate. However, the benefits of understanding and using non-verbal communication extend into the classroom. Within an educational setting or teaching context, it is important for law teachers to understand non-verbal communication and its relevance to sustained learning (York "Non-Verbal Immediacy's Role in Student Learning" 2015 Journal of Media and Communication Studies 7). Studies have shown that the non-verbal component of classroom communication is more important than the verbal 
component and all teachers should be aware of the possible influences of non-verbal behaviours on learners, the learning environment and eventually practice (Smith "Nonverbal Communication in Teaching" 19794 Review of Educational Research 631-672).

As illustrated earlier, the ability to decode non-verbal communication is crucial to effective lawyering, and all law students should be trained in this unheeded form of communication. The natural, unrehearsed nature of nonverbal communication makes it a thought-provoking, yet fascinating, field to study. Technology in the form of video recorders, digital cameras, smartphones and computers has made it possible to view, analyse and decode non-verbal behaviour from a scientific angle (Bartlett, Hager, Ekman and Sejnowski "Measuring Facial Expressions by Computer Image Analysis" 199936 Psychophysiology 253). The use of such technology has drastically improved the accuracy and reliability of non-verbal measuring with some experiments and tests showing an accuracy of over 90 per cent (Bartlett et al 1999 Psychophysiology 253).

It is important for any teacher to understand the dynamics and operations of non-verbal communication before exposing students to this dynamic form of communication (Bambaeeroo and Shokpour "The Impact of the Teachers' Nonverbal Communication on Success in Teaching" 2017 Journal of Advances in Medical Education and Professionalism 51). As a starting point, a teacher could record a teaching lesson and analyse the types of message being sent to the students. The teacher could also analyse the students' reactions and behaviour in the classroom as a means of improving the total communication process (Kundu 1976 Educational Technology 31-33). A mirror could also be used as an effective tool for teachers to analyse and become aware of their own non-verbal cues emitted in a classroom setting. The ability to motivate students by using non-verbal cues can be crucial to developing interest in the subject matter as well as to facilitating active learning. Becoming aware of non-verbal signs that convey negative and positive messages to the student in the classroom is vital to improving student-teacher relationships and interaction in the learning environment.

As part of skills training, law students could view recordings of real-life trials where facial expressions and body language of the various role-players in court are analysed. This could be a better method of analysis than attaching recording equipment to the bodies of students, which could spark a self-conscious reaction resulting in non-verbal communication becoming restricted. A simulation or recoding of a consultation between a client and lawyer within the parameters of ethical considerations can provide rich information on the essence of non-verbal communication. According to Bull, the practical significance and impact of studying non-verbal communication far surpasses formal training procedures (Bull 2001 The Psychologist 644). An appreciation of communication is possible through the dissection of books, articles and electronic media but key to understanding non-verbal communication is to accept, absorb, experiment and to become aware of its role in the law school curriculum. 


\section{$6 \quad$ Conclusion}

Although one cannot rely on non-verbal cues alone in every legal setting, it has been established that awareness of these cues can provide valuable information and offer an improved skill-set for any lawyer (Pease Body Language 57). According to the South African Qualification Standard for Bachelor of Laws (Council of Higher Education 2015) "graduates should thus be able to acknowledge and appreciate linguistic diversity, and programmes leading to the LLB ought to take this need into account, in order to prepare graduates to practise law competently in a context of such diversity". It is therefore imperative that law schools become creative and address the disjuncture between legal education, the professional demands and the shortage of practice skills that exist among law graduates. For those schools and curriculums that do not have non-verbal communication as a standalone module at this stage, a slight tweak of the existing curriculums could see non-verbal communication incorporated into important practical modules such as Legal Skills or Legal Practice and even Introduction to Law, among others. In fact, a component of non-verbal communication could be introduced into most law modules. However, the prospect of training lawyers to become more knowledgeable of non-verbal cues is still a process in the making (Shadow "Improving Nonverbal Communication in Criminal Justice" 2008 Law 5). For the present, a greater alertness to occasions of non-verbal proceedings is paramount to improving communication in the legal process (Galloway "Nonverbal Communication" 1968 Theory into Practice 172). Research on non-verbal communication has shown that it does improve the communication process as well as assist people to understand themselves and others (Burgoon et al Nonverbal Communication: The Unspoken Dialogue 27). For the legal profession, an understanding of the dynamics of non-verbal communication is vital to improving the overall legal communication skills of the attorney (Kevelson The Law as a System of Signs 5). The need to make non-verbal communication part and parcel of every law curriculum has never been greater.

Desan lyer University of Zululand 